\title{
FAST SOLAR FLARE PROTON ACCELERATION BY MHD TURBULENCE
}

\author{
DEAN F. SMITH \\ Berkeley Research Associates \\ and Department of Astrophysical, Planetary, and Atmospheric Sciences \\ University of Colorado, Boulder \\ Boulder, Colorado 80309
}

\begin{abstract}
Proton acceleration by short-wavelength Alfven (A) waves resonant at the first harmonic of the proton gyrofrequency is reconsidered, taking into account nonlinear wave-wave interactions, collisionless wave losses, and wave escape losses in the geometry of a model coronal loop. It is shown that for the A wave levels required for acceleration in the transrelativistic regime in the 1982 June 3 flare and for acceleration in the nonrelativistic regime in the 1980 June 7 flare, the nonlinear wave interaction of scattering on the polarization clouds of ions will be important. This interaction rapidly isotropizes the $\mathrm{A}$ waves which divide their energy with fast magnetosonic (M) waves with a negligible change in their frequency spectrum. Because of electron Landau damping and escape losses, the $M$ waves are confined to two narrow cones about the magnetic field and the total $(A+M)$ wave distribution is still highly anisotropic. The total $(\mathrm{A}+\mathrm{M})$ wave spectrum has the same acceleration efficiency as a pure $\mathrm{A}$ wave spectrum. There are two principal problems with models of this type. The first is that a large wave energy density is required in a fairly narrow range in $\mathbf{k}$-space. The second is that the protons are effectively bottled up. This makes very impulsive behavior as in the 7 June 1980 flare difficult to explain because proton precipitation is relatively slow.
\end{abstract}

\section{Introduction}

Stochastic acceleration of ions by resonant Alfven waves is a viable mechanism with the restriction that there must be appreciable power in Alfven waves propagating both parallel and antiparallel to the magnetic field B. The basic paper on this process is Barbosa (1979). An extension of his results to the nonlinear regime appeared in Smith and Brecht (1989; hereafter SB). We review and extend these results here.

Miller and Ramaty (1987; hereafter MR) performed a Monte-Carlo simulation to extend the analytic results of Barbosa (1979) into the transrelativistic regime to explain the 1982 June 3 flare. Gamma-ray observations (Chupp et al. 1987) show that the 4.1-6.4 MeV emission due to 30 $\mathrm{MeV}$ protons and the pion emission due to $\sim 1 \mathrm{GeV}$ protons peaked simultaneously to within $16 \mathrm{~s}$ at $\sim 11: 43.5 \mathrm{UT}$. This simultaneity implies an acceleration time $\tau_{\mathrm{A}}$ from $\sim 30 \mathrm{MeV}$ to $1 \mathrm{GeV}$ less than $16 \mathrm{~s}$ which leads to a total energy density in Alfven turbulence $\mathrm{W}^{\mathrm{A}}>2.3 \mathrm{erg} \mathrm{cm}^{-3}$ according to MR. Although this $W^{A}$ is a small fraction of the energy density in a 100 or $300 \mathrm{G}$ field, it is comparable to the thermal energy density for $\mathrm{n}=5 \times 10^{10} \mathrm{~cm}^{-3}, \mathrm{~T}_{\mathrm{e}}=\mathrm{T}_{\mathrm{i}}=1.6 \times 10^{6} \mathrm{~K}$. This relatively high value of $\mathrm{W}^{\mathrm{A}}$ leads to the possibility of nonlinear interactions in which Alfven waves $A$ are transformed into other Alfven waves $A^{\prime}$ or magnetosonic waves $M$ and vice versa. Such interactions could lead to additional losses since $M$ waves are more heavily damped than $A$ waves. The most effective nonlinear interaction is scattering on the polarization clouds of ions which was considered by Livshits and Tsytovich (1970) and Kaplan and Tsytovich (1973). These processes can be represented schematically by 


$$
\begin{aligned}
& A+i \leftrightarrows A^{\prime}+i^{\prime} \\
& A+i \leftrightarrows M+i^{\prime}, \\
& M+i \leftrightarrows M^{\prime}+i^{\prime}
\end{aligned}
$$

where $i$ and $i$ represent the polarization cloud of the ion before and after the scattering respectively.

It turns out for cases of interest that the $M$ waves are confined to fairly narrow cones along the direction of $\mathbf{B}$ (z-axis) so that the primary component of the wavevector $\mathbf{k}$ is $\mathbf{k}_{\mathbf{z}}$. Under these conditions the change in angular frequency $\omega$ for processes $(1 a, b, c)$ is negligible and the change in $k_{Z}$ is very small. This is fortunate because the assumed spectral form of Alfven waves $W^{A}(\omega)$ will not be significantly affected by these processes and the spectrum of magnetosonic waves $\mathrm{W}^{\mathrm{M}}(\omega)$ is known from the specification of $\mathrm{W}^{\mathrm{A}}(\omega)$. Along with $\mathrm{MR}$ we assume that $\mathrm{W}^{\mathrm{A}}(\omega)$ has a Kolmogorov spectrum $\sim \omega^{-573}$ which has some justification in the measurements of Matthaeus and Goldstein (1982). However, measurements from $\omega^{-1}$ to $\omega^{-2}$ have also been made (Sari and Valley 1976) and a variety of theoretical predictions have been given (Montgomery 1983).

Besides the possibility of enhanced losses due to nonlinear processes, there are wave escape losses in the geometry of a solar loop. The interaction region for waves and protons has a finite spatial extent which leads to losses for both $A$ and $M$ waves given in SB. The $M$ waves are also subject to collisionless Landau damping by electrons at a rate given in SB. For simplicity we assume that the magnetic field lines follow a semicircular loop of length $L$ and minor radius $R$, and that there are no currents flowing along $B$. We neglect convergence of magnetic field lines in the acceleration region which is assumed to be in the corona.

We summarize some results on the total energy in protons and their energy distribution to set limits on the volumes of the acceleration region. The proton distribution in a hard sphere scattering model is characterized by the parameter $\alpha \mathrm{T}$, a measure of the hardness of the distribution in a Bessel function fit (Ramaty and Murphy 1987). Here, $\alpha=V^{2} \lambda c$, where $V$ is the velocity of the scatterers and $\lambda$ is the scattering mean free path. As explained in Ramaty (1986) and Hua and Lingenfelter (1987; hereafter HL) it is possible to determine $\alpha \mathrm{T}$ and the number of protons $\mathrm{N}_{\mathrm{p}}$ above a threshold energy, usually taken as $30 \mathrm{MeV}$, from the ratio of the prompt 4-7 MeV $\gamma$-ray fluence to the delayed $2.223 \mathrm{MeV} \gamma$-ray fluence. For example, for the 1980 June 7 flare HL derived $\alpha \mathrm{T}=0.021 \pm 0.003$ and $\mathrm{N}_{\mathrm{p}}(>30 \mathrm{MeV})=(9.2 \pm 2.4) \times 10^{31}$. This leads to a total energy above $1 \mathrm{MeV}$ of $\sim 2 \times 10^{29} \mathrm{erg}$.

The time over which there was significant $\gamma$-ray production was $50 \mathrm{~s}$ (Chupp 1983). Hence, $4 \mathrm{x}$ $10^{27} \mathrm{erg} \mathrm{s}^{-1}$ was going into protons. Because the 1982 June 3 flare consisted of an impulsive phase followed by a more gradual phase due to shock acceleration, it is not possible to make deductions of comparable accuracy since the published number of protons $\mathrm{N}_{\mathrm{p}}$ refers to the whole flare. However, approximately $\mathrm{N}_{\mathrm{p}}(>30 \mathrm{MeV})=3 \times 10^{32}$ and $\alpha \mathrm{T}=0.035 \pm 0.003$ for the impulsive phase (HL) which leads to a total energy above $1 \mathrm{MeV}$ of $\sim 7 \times 10^{29} \mathrm{erg}$. The impulsive phase lasted $66 \mathrm{~s}$ for this flare. Hence, $1.1 \times 10^{28} \mathrm{erg} \mathrm{s}^{-1}$ was going into protons. Although the region in which this energy is processed could be smaller as in reconnection models of flares, we will minimize wave escape losses by taking a volume of $10^{27} \mathrm{~cm}^{3}$ and finding $L$ and $R$. The volume of a loop is $\pi \mathrm{L}^{3}(\mathrm{R} / \mathrm{L})^{2}$. Typical $\mathrm{R} / \mathrm{L}$ values are $10 \%$ which leads to $\mathrm{R}=3.2 \times 10^{8} \mathrm{~cm}, \mathrm{~L}=$ $3.2 \times 10^{9} \mathrm{~cm}$, and a loop height $\sim 10^{9} \mathrm{~cm}$. Smaller loop heights can be obtained in a multiple loop model, but SB give evidence that a single loop was involved in the 1980 June 7 flare. Thus, we limit the scope of this paper to developing a single loop model in as much detail as possible. 


\section{Model and Wave Results}

The model consists of a semicircular loop with a uniform toroidal field in which Alfven waves initially propagating along the field are excited. Further details of the model are given in SB. All that is important for our purposes is that the waves have an energy density $\mathrm{W}^{\mathrm{A}}$.

To obtain a level $\mathrm{W}^{\mathrm{A}}$ for waves in the 1980 June 7 flare necessary to accelerate protons to 30 $\mathrm{MeV}$ in $2 \mathrm{~s}$ (Chupp 1983), we use a formula for the acceleration time $\tau_{\mathrm{A}}$ due to Melrose (1974) which was also obtained by Barbosa (1979)

$$
\tau_{\mathrm{A}}=\left\{\frac{\pi}{4} \omega\left(\frac{\delta \mathrm{B}}{\mathrm{B}}\right)^{2} \frac{\mathrm{v}_{\mathrm{A}}}{\mathrm{v}}\right\}^{-1},
$$

where $\delta B$ is the perturbation in $B$ due to the presence of the waves. Thus, $(\delta B)^{2} / 8 \pi=W^{A}$, or more generally, $W^{A}+W^{M}$, where $W^{M}$ is the energy density in $M$ waves. With $\tau_{A}=2 \mathrm{~s}, \omega=1.3$ $\times 10^{4} \mathrm{~s}^{-1}, \mathrm{v}_{\mathrm{A}}=10^{8} \mathrm{~cm} \mathrm{~s}^{-1}$ and $\mathrm{v}=7.6 \times 10^{9} \mathrm{~cm} \mathrm{~s}^{-1}$ corresponding to a $30 \mathrm{MeV}$ proton, $\delta \mathrm{B} / \mathrm{B}=$ 0.062 which leads to $\mathrm{W}^{\mathrm{A}}=1.5 \mathrm{erg} \mathrm{cm}^{-3}\left(\mathrm{~W}^{\mathrm{M}} \approx 0\right.$ initially).

An important problem for the amplification processes that will be considered next is the initial level of $\mathrm{M}$ waves, $\mathrm{W}_{\mathrm{o}}^{\mathrm{M}}\left(\omega_{\mathrm{min}}\right)$. It is reasonable to suppose that no wave excitation mechanism will excite purely Alfven waves. Alternately, even in the case of pure A wave excitation, weak density gradients would lead to a low level of $M$ waves (Wentzel 1989). Hence, we shall assume that $0.1 \%$ of the energy going into Alfven waves goes into $M$ wave excitation and $A$ wave excitation at other angles with the same spectral form. This implies $\mathrm{W}_{\mathrm{O}}^{\mathrm{M}}=\mathrm{W}_{\mathrm{O}}^{\mathrm{A}^{\prime}}=2.3 \times 10^{-3}\left(1.5 \times 10^{-3}\right) \mathrm{erg}$ $\mathrm{cm}^{-3}$ for the 1982 June 3 (1980 June 7) flare.

The equations describing the nonlinear interaction of $A$ and $M$ waves due to scattering on the polarization clouds of ions are given in SB. We concentrate on the results here. It follows from these equations that two A-waves with wave vectors in the same half-plane do not interact. Likewise, there is no interaction between two $M$ waves propagating in the same direction. A waves amplify $A$ and $M$ waves propagating in the other half-plane of the same $\omega$ at a rate

$$
v_{n l}=\frac{\pi}{3} \frac{\omega^{2} W_{\omega}^{A}}{n_{i} v_{A}{ }^{2}\left(1+T_{e} / T_{i}\right)^{2}} .
$$

The coefficient in equation (3) is valid for arbitrary distributions of $A$ waves and for $M$ waves with

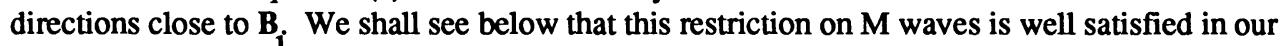
applications. If $v_{\mathrm{nl}}^{-1}$ is small compared to time scales of interest so that several e-folds of amplification can occur, then initially one-dimensional $A$ waves become almost isotropic. There is a very small wedge of $\lesssim \pm 0.5^{\circ}$ around $\theta=90^{\circ}$ where A waves are Landau damped and do not grow. For the same condition, $M$ waves grow up to a level where $\mathrm{W}^{\mathrm{M}}=\mathrm{W}^{\mathrm{A}}$ in two cones about $\mathrm{z}$

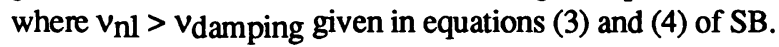

Application of these ideas leads to the results in Table 1 from SB where $v_{g}=v_{n l}-v_{\text {damping }}$ is the net nonlinear growth rate and $\tau$ is the time for $6.2 \mathrm{e}$-folds of growth, the time for $M$ waves to grow to half the initial level. This is the saturation level since energy is now divided equally between $\mathrm{A}$ and $\mathrm{M}$ waves. 
TABLE 1. Total and net nonlinear growth rates

For both flares $\mathrm{n}=4.8 \times 10^{10} \mathrm{~cm}^{-3}$ and $\mathrm{T}_{\mathrm{e}} / \mathrm{T}_{\mathrm{i}}=1$

\begin{tabular}{lcccccc}
\hline Flare & $\omega_{\min }\left(\mathrm{s}^{-1}\right)$ & $\mathrm{W}_{\omega}^{\mathrm{A}}\left(\mathrm{erg} \mathrm{cm}^{-3} \mathrm{~s}\right)$ & $\mathrm{v}_{\mathrm{A}}\left(\mathrm{cm} \mathrm{s}^{-1}\right)$ & $v_{\mathrm{nl}}\left(\mathrm{s}^{-1}\right)$ & $v_{\mathrm{g}}\left(\mathrm{s}^{-1}\right)$ & $\tau(\mathrm{s})$ \\
\hline & & & & & & \\
1982 June 3 & $2.8 \times 10^{4}$ & $5.4 \times 10^{-5}$ & $3 \times 10^{8}$ & 1.5 & 1.4 & 4.4 \\
1980 June 7 & $1.3 \times 10^{4}$ & $7.9 \times 10^{-5}$ & $10^{8}$ & 4.2 & 4.17 & 1.5 \\
\hline
\end{tabular}

TABLE 2. Angular range and average damping of $M$ waves For both flares $\mathrm{ve}_{\mathrm{e}}=4.9 \times 10^{8} \mathrm{~cm} \mathrm{~s}^{-1}$

\begin{tabular}{lllll}
\hline Flare & $\omega_{\min }\left(\mathrm{s}^{-1}\right)$ & $\mathrm{v}_{\mathrm{A}}\left(\mathrm{cm} \mathrm{s}^{-1}\right)$ & $\theta_{1}$ & $v^{\mathrm{M}}\left(\mathrm{s}^{-1}\right)$ \\
\hline & & & & \\
1982 June 3 & $2.8 \times 10^{4}$ & $3 \times 10^{8}$ & $18.7^{\circ}$ & 0.82 \\
1980 June 7 & $1.3 \times 10^{4}$ & $10^{8}$ & $25.1^{\circ}$ & 2.1 \\
\hline
\end{tabular}

It can be seen from the values of $\tau$ in Table 1 that nonlinear effects will be important in the first $16 \mathrm{~s}$ interval for the 1982 June 3 flare. Nonlinear effects will be marginally important in the first $2 \mathrm{~s}$ of the 1980 June 7 flare. The impulsive phase $\gamma$-ray emission of this flare lasts $50 \mathrm{~s}$. Thus, nonlinear effects will certainly be important for most of the $\gamma$-ray emission to the extent that all the emission comes from one loop.

The angular range of the $M$ waves and their average damping were calculated in SB and are given in Table 2 , where $\theta_{1}$ is the half-angle of the cone along $\mathbf{B}$ to which $\mathbf{M}$ waves are confined because of electron Landau damping.

The following general picture emerges for the parameters of these two flares. Nonlinear effects will be important for most of the impulsive phases of both flares to the extent that all the emission comes from a single loop. The initial $A$ waves will be rapidly isotropized. The $M$ waves will grow in two cones aligned along the field where their damping is sufficiently weak to a level with $W M_{\approx}$ $\mathrm{W}^{\mathrm{A}}$. For both flares the principal loss is thermal Landau damping of $M$ waves. We shall see in the next section that these losses are comparable to the energy leaving the acceleration region in accelerated protons.

\section{Proton Acceleration}

The overall acceleration picture follows closely the one of Barbosa (1979) with the caveat that one of the wave modes ( $M$ waves) are subject to other forms of damping. The process (1a) preserves $\omega^{A}$ which means that it preserves $k_{z}^{A}=k^{A} \cos \theta$. Thus, larger (smaller) values of $k^{A}$ are created in the scattering process with momentum being obtained from (given to) the polarization cloud of the ion. An initial one-dimensional $\mathrm{k}_{\mathrm{z}}-5 / 3$ spectrum with $\mathrm{k}_{\mathrm{min}}$ does not lead to an isotropic $\mathrm{k}^{-5 / 3}$ spectrum for which the minimum $k=0$. By taking $|\cos \theta|=2 / \pi$, MR effectively took a onedimensional (1-D) spectrum at $\theta=50.4^{\circ}$ which differs from the spectrum of Section 2 by the scale factor $\pi / 2$. In process (1b), the $k_{z}^{A}$ of the $A$ wave becomes the $k^{M}$ of the $M$ wave since frequency 
is preserved. Since the $M$ waves are concentrated in narrow cones about $\mathrm{z}$ with most of the power close to $\theta=0$, the $\mathrm{k}_{\mathrm{Z}}$ spectrum of these waves is $\mathrm{k}_{\mathrm{Z}}-5 / 3$ to within an error of less than $5 \%$ as long as the $\mathrm{M}$ waves are in a cone of half-angle $\leq 26^{\circ}$. Considering the degree of uncertainty of other parameters in the problem, it is reasonable to disregard this small error and write the resonance condition as (cf. MR)

$$
\omega=\Omega_{\mathrm{i}} \mathrm{v}_{\mathrm{A}} / \gamma \mathrm{v}|\cos \phi|
$$

for all the waves, $A$ or $M$, where $v$ is the velocity and $\phi$ the pitch angle of the proton, respectively, $\Omega_{\mathrm{i}}=\mathrm{eB} / \mathrm{m}_{\mathrm{i}} \mathrm{c}$ is the nonrelativistic gyrofrequency and $\gamma$ is the proton's Lorentz factor.

With the same resonance condition (4) and the same $\omega$ spectrum, the $M$ waves have exactly the same acceleration properties as A waves, but the physical picture is slightly changed. The A wave is left-hand circularly polarized and the $M$ wave is right-hand circularly polarized with the perturbation electric field $90^{\circ}$ out of phase with that of the A wave. Because of the gyromotion of a proton, the same average force components are present as for an A wave and the proton can gain or lose energy depending on its cyclotron phase. An average over a random-phased, isotropic distribution of protons leads to cyclotron damping of the $\mathbf{M}$ wave. Just as for the A wave, scattering is a stronger process than acceleration and the isotropy of the proton distribution is automatically insured by the acceleration.

The injection problem of having some protons with $v>v_{A}$ remains. As discussed by $S B$, this problem is tied to the flare primary energy release and the source of the $A$ or $A$ and $M$ waves. A loop with many tearing modes occurring in a small fraction of the total volume is a good candidate which leads to the acceleration of a small fraction $(\sim 1 \%)$ of the ions to $v>v_{A}$. The figures for energy above $1 \mathrm{MeV}$ in Section 1 with a volume of $10^{27} \mathrm{~cm}^{3}$ lead to the requirement of accelerating $0.1-1 \%$ of the protons to $v>v_{A}$ throughout the whole impulsive phase. This is consistent with the picture of tearing modes which instantaneously occur in a small fraction of the volume, but which sequentially cover or process the magnetic energy of a large fraction of the volume.

We consider the energetics of the process with $A$ and $M$ waves as compared to the pure $A$ wave result. As given in Section 1, for the 1982 June 3 flare there is $11 \mathrm{erg} \mathrm{cm}^{-3} \mathrm{~s}^{-1}$ going into proton energy above $1 \mathrm{MeV}$. We found in Section 2 an average loss rate due to $M$ waves $v^{M}=0.82 \mathrm{~s}^{-1}$. With $\mathrm{WM}^{\mathrm{M}}=1.2 \mathrm{erg} \mathrm{cm}^{-3}$ (half of $2.3 \mathrm{erg} \mathrm{cm}^{-3}$ which was the initial level), there is an additional loss of $1.0 \mathrm{erg} \mathrm{cm}^{-3} \mathrm{~s}^{-1}$, about $9 \%$ of the proton energy loss. For the 1980 June 7 flare there is 4 erg cm $\mathrm{cm}^{-3} \mathrm{~s}^{-1}$ going into proton energy above $1 \mathrm{MeV}$ (cf. §I). We found in Section 2 an average loss rate due to $M$ waves $v^{M}=2.1 \mathrm{~s}^{-1}$. With $\mathrm{W}^{\mathrm{M}}=0.75 \mathrm{erg} \mathrm{cm}^{-3}$ (half of $1.5 \mathrm{erg} \mathrm{cm}^{-3}$, the initial level), there is an additional loss of $1.6 \mathrm{erg} \mathrm{cm}^{-3} \mathrm{~s}^{-1}$, about $40 \%$ of the proton energy loss. Hence, these losses are of the same order of magnitude as the accelerated proton losses and should be included in any detailed energetic considerations. They are much larger than the pure A wave case.

\section{Discussion}

We have reexamined proton acceleration by short-wavelength Alfven waves resonant at the first harmonic of the proton gyrofrequency. Within the limitations of the assumptions made such as a uniform field in a semicircular loop, the results are the following. For levels of waves which have already been proposed for proton acceleration in the transrelativistic regime (MR) and necessary for a well known impulsive flare in the nonrelativistic regime, initially 1-D A waves will rapidly 
become almost isotropic and give half their energy to almost 1-D $M$ waves. This result partially justifies MR's use of an isotropic distribution of $A$ waves. However, the total distribution of $A$ and $M$ waves remains peaked along $z$ due to the almost 1-D $M$ wave angular distribution. Important results of SB are the demonstration of the importance of $M$ waves and the identification of two substantial loss mechanisms for these waves, escape losses and thermal electron Landau damping losses. We have not considered variations in the initial A wave spectrum, but believe that spectra in the observed range from $\omega^{-1}$ to $\omega^{-2}$ (Sari and Valley 1976) would lead to similar results.

Although this type of model is promising, there are two problems which deserve mention. The first problem is that there is a large energy density $W^{A}+W^{M}$ in a fairly narrow range of $\omega$ - or $k$ space. There will be another potentially large loss due to cascading from small to large $\mathbf{k}$. Unfortunately, our knowledge of MHD turbulence is sufficiently poor (Montgomery 1983) that there is no way to meaningfully evaluate this loss. We are simply assuming that acceleration occurs in part of the inertial range of MHD turbulence and that the acceleration does not significantly affect this range.

The second problem is that the protons are well confined by the levels of turbulence required for acceleration and the proton transport is diffusive. The mean free path for scattering is (Forman, Ramaty, and Zweibel 1986)

$$
\lambda_{s}=\frac{m_{i}^{2} v^{2} c^{2}}{12 \pi^{2} e^{2} w\left(k_{R}\right)}
$$

where the resonant wavenumber $k_{R}$ is given by the right hand side of equation (4) without $V_{A}$ and

$$
\mathrm{W}^{\mathrm{A}}+\mathrm{W}^{\mathrm{M}}=\int \mathrm{W}(\mathrm{k}) \frac{\mathrm{dk}}{2 \pi} .
$$

As discussed by Barbosa (1979) the time for the protons to diffusively travel a loop half length $L / 2$ is

$$
\tau_{\mathrm{d}} \cong \frac{\mathrm{L}^{2}}{4 \kappa_{\|}}
$$

where the parallel spatial diffusion coefficient

$$
\kappa_{\|}=\frac{2}{3} v \lambda_{\mathrm{s}}=\frac{\mathrm{m}_{\mathrm{i}}^{2} \mathrm{v}^{3} \mathrm{c}^{2}}{18 \pi^{2} \mathrm{e}^{2} \mathrm{~W}\left(\mathrm{k}_{\mathrm{R}}\right)} .
$$

Results of using equations (5) and (8) for the parameters given above are given in Table 3 where the first two rows are for the flare of 1980 June 7 and the last two rows are for the flare of 1982 June 3.

TABLE 3. Proton Mean Free Paths and Propagation Times

\begin{tabular}{lllcc}
\hline $\mathrm{E}(\mathrm{MeV})$ & $\mathrm{v}\left(\mathrm{cm} \mathrm{s}^{-1}\right)$ & $\lambda_{\mathrm{s}}(\mathrm{cm})$ & $\tau_{\mathrm{d}}(\mathrm{s}) ; \mathrm{L}=10^{9} \mathrm{~cm}$ & $\tau_{\mathrm{d}}(\mathrm{s}) ; \mathrm{L}=10^{8} \mathrm{~cm}$ \\
\hline & & & & \\
1 & $1.4 \times 10^{9}$ & $5.9 \times 10^{4}$ & $4.7 \times 10^{3}$ & 47 \\
30 & $4.4 \times 10^{9}$ & $1.0 \times 10^{5}$ & $8.3 \times 10^{2}$ & 8.3 \\
30 & $4.4 \times 10^{5}$ & $5.2 \times 10^{4}$ & $1.7 \times 10^{3}$ & 17 \\
$10^{3}$ & $3.0 \times 10^{10}$ & $8.3 \times 10^{4}$ & $1.5 \times 10^{2}$ & 1.5 \\
\hline
\end{tabular}


It can be seen that these times are too long to explain the most rapid (2s) variations in the 1980 June 7 flare and possible for the 1982 June 3 flare only for short $\left(\mathrm{L}=10^{8} \mathrm{~cm}\right)$ acceleration region lengths.

It is in principle possible to circumvent this problem by going to very short acceleration region lengths, but then many acceleration regions or a single very large area region is required. The latter possibility is inconsistent with current knowledge about loop $R / L$ values. The problem with many acceleration regions is that an additional coordinating mechanism is required to produce the semiperiodic behavior that was observed in the 1980 June 7 flare.

Neither of the above problems is insurmountable and both problems should be considered as further restrictions on the use of MHD turbulence models.

This work was supported by NSF Grant ATM-8909845.

\section{References}

Barbosa, D.D.: 1979, Ap. J. 233, 383.

Chupp, E.L.: 1983, Solar Phys. 86, 383.

Chupp, E.L. et al.: 1987, Ap.J. 318, 913.

Forman, M.A., Ramaty, R., and Zweibel, E.G. 1986, in Physics of the Sun, Vol. II., ed. P.A. Sturrock (Dordrecht: Reidel), p. 245.

Hua, X.-M., and Lingenfelter, R.E.: 1987, Solar Phys. 107, 351 (HL).

Kaplan, S.A., Tsytovich, V.N.: 1973, Plasma Astrophysics, trans. D. ter Haar (Oxford: Pergamon Press), p. 68.

Livshits, M.A., and Tsytovich, V.N.: 1970, Nuclear Fus. 10, 241.

Matthaeus, W.H., and Goldstein, M.L.: 1982, J. Geophys. Res. 87, 6011.

Melrose, D.B.: 1974, Solar Phys. 37, 353.

Miller, J.A., and Ramaty, R.: 1987, Solar Phys. 113, 195 (MR).

Montgomery, D.: 1983, in Solar Wind Five, ed. M. Neugebauer, NASA CP-2280, p. 107.

Ramaty, R.: 1986, in Physics of the Sun, Vol. II., ed. P.A. Sturrock (Dordrecht: Reidel), p. 251.

Sari, J.W., and Valley, G.C.: 1976, J. Geophys. Res. 81, 5489.

Smith, D.F., and Brecht, S.H.: 1989, Ap. J. 344, 1004 (SB).

Wentzel, D.G.: 1989, Ap. J. 336, 1073.

\section{DISCUSSION}

STEPANOV: What is the origin of Alfvén wave turbulence in a flare loop?

SMITH: I have not considered this problem. I have simply assumed that the Alfvén waves are there.

HOYNG: How sensitive are your results to the assumed spectral distribution of waves $\left(k^{-5 / 3}\right)$ ? 
SMITH: I have only done calculations for the range $\mathbf{k}^{-1}$ to $\mathbf{k}^{-2}$ observed by Sari and Valley in the solar wind. Within this range the results are not sensitive to the assumed spectral distribution.

SIVARAM: (i) Would this process also accelerate other ions? One has a preferential acceleration mechanism for $\mathrm{He}-3$ for instance?

(ii) Could the protons be accelerated to energies well over a GeV?

(iii) As neutrons are produced, would tritium form from $D$.

SMITH: (i) I have not investigated this question, but one could have resonant acceleration of other ions by Alfvén waves, but of different frequencies.

(ii) If you extend the spectrum to lower frequencies, protons could be accelerated above 1 GeV.

(iii) The cross-section for this process for solar densities is too small to be important. 